\title{
Total electron scattering cross section from sevoflurane by 1-300 eV energy electron impact
}

\author{
A. I. Lozano, ${ }^{1,2}$ F. Ferreira da Silva, ${ }^{3}$ F. Blanco, ${ }^{4}$ P. Limão-Vieira ${ }^{3}$ and G. García ${ }^{1 *}$ \\ ${ }^{1}$ Instituto de Física Fundamental, Consejo Superior de Investigaciones Científicas (CSIC), Serrano 113-bis, 28006 \\ Madrid, Spain \\ ${ }^{2}$ Escuela de Doctorado de la UNED-Programa de Doctorado en Ciencias, 28015 Madrid, Spain \\ ${ }^{3}$ Atomic and Molecular Collisions Laboratory, CEFITEC, Department of Physics, Universidade NOVA de Lisboa, \\ 2829-516, Caparica, Portugal \\ ${ }^{4}$ Departamento de Fisica Atomica, Molecular y Nuclear, Facultad de Ciencias Fisicas, Universidad Complutense de \\ Madrid, E-28040 Madrid, Spain
}

\begin{abstract}
We report on novel total electron scattering cross section (TCS) measurements for electrons scattering from sevoflurane, at incident electron impact energies in the range 1-300 eV. The experimental results, obtained from a newly implemented magnetic beam apparatus based in Madrid, are compared with theoretical results from the independent atom model with screening corrected additivity rule including interference effects and rotational excitation (IAM-SCAR+I+R). A very good level of agreement has been found between the present experimental and theoretical data at above about $20 \mathrm{eV}$ electron impact and to within the experimental uncertainties.

\footnotetext{
* Corresponding author.

E-mail address: g.garcia@csic.es (G. García).
} 


\section{Introduction}

Since the first use of ether as a general anaesthetic in 1842 by Crawford Williamson Long, inhaled anaesthetics have been widely used in surgical practice. The international community has been focused in understanding the role of such chemical compounds within the physiological environment, however how a given drug reversibly alters the central nervous system function still remains poorly understood. Currently, it is known that inhaled anaesthetics can modify the activity of a wide variety of proteins [1-4] by binding to discrete sites. Such knowledge has changed the traditional notion that all general anaesthetics act non-specifically. Thus, focusing on a knowledge of the molecular structure and chemical properties of these molecules may further help our understanding of their molecular reactivity and thereby ultimately improve the clinical utility of general anaesthetics. In this study we have focused on the important halogenated inhaled anaesthetic, sevoflurane (Fig. 1), which was introduced into clinical practice in the 1990s. Nowadays, it is one of the most used for induction and maintenance of general anaesthesia in different types of surgery and is also commonly used in children's clinical practice as well as in veterinary use. Sevolflurane possesses properties that are well attuned to an inhalation anaesthetic agent, due to its low solubility in blood in comparison with other inhaled anaesthetics like halothane and isoflurane [5].

An $a b$ initio study, using both restricted Hartree-Fock (RHF) and hybrid B3LYP DFT quantum chemistry methods, to investigate the structure, charge distribution and electric dipole moment of sevoflurane $\left(\mathrm{C}_{4} \mathrm{H}_{3} \mathrm{~F}_{7} \mathrm{O}\right)$ has been performed by Tang et al. [6]. These authors reported that even within the same $-\mathrm{CF}_{3}$ group, the RHF and DFT bond lengths and angles are not kept identical due to the overall molecular asymmetry which also causes a complex charge distribution. The structure of the anaesthetic haloether sevoflurane has been determined using Fourier-transform microwave spectroscopy, where complementary Stark effect measurements obtained its electric dipole moment to be 2.27D [7]. Vibrational assignments have been reported by Dom et al. [8] in infrared spectroscopy experiments together with several sevoflurane/benzene complexes. These complexes have interactions which are interesting from the chemical and biological points of view, since they may serve to model local interactions of protein receptors with aromatic side-chain molecules [9]. Langbein et al. [10] reported on volatile anaesthetics and their relevance to atmosphere chemistry, together with the role of chlorofluorocarbons (CFC) and halons in damaging the stratospheric ozone layer. Finally, we have recently explored by means of experimental and theoretical methods, 
the elastic differential and integral cross sections, for electrons scattering from sevoflurane in the 10-50 eV impact energy range [11].

In this paper, we include experimental data of total electron scattering cross sections from sevoflurane in the energy range 1-300 eV using a magnetically confined experimental system [12]. Those experimental data are compared with results from our ab initio independent atom modelscreening-corrected additivity rule with interference and rotational terms (IAM-SCAR+I+R) method [13-15]. Excellent agreement is found between them at energies above about $20 \mathrm{eV}$. The remainder of the present paper is structured as follows. In Sec. II, following this introduction, we provide a brief description of the experimental configuration and the theoretical methodology. In Sec. III we present and discuss our experimental and theoretical results. Finally, in Sec. IV, some conclusions from the present work are drawn.

\section{Experimental and theoretical methods}

\section{A. Experimental setup}

Figure 2 shows a schematic diagram of the experimental setup which has been developed and implemented at CSIC, Madrid, and which is intended to perform low energy electron scattering from molecular targets using a strong confining axial magnetic field. A thorough description of the main working principles and experimental details has been given recently $[12,16]$. Briefly, it is based on the magnetic confinement of an electron beam that passes through a gas trap and a scattering chamber prior to its detection by an MCP. Such a strong magnetic field $(\sim 0.1 \mathrm{~T})$ ensures that the electron's path is not affected by external fields (such as the earth's magnetic field), in particular at low kinetic energies. The electron kinetic energy $(E)$ within a magnetic field can be separated into the parallel $(\|)$ and perpendicular $(\perp)$ components of the velocity, relative to the axial field direction (gyromotion). As described in Ref. [12] under these axial magnetic confinement conditions, any collision event in the gas trap/scattering chamber converts the expected scattering angle $(\theta)$ into a kinetic energy loss in the direction parallel to the beam $\left(\mathrm{E}_{\|}\right)$, according to $\mathrm{E}_{\|}=\mathrm{E}_{\|} \cos ^{2} \theta$. As clearly denoted in figure 2, the system comprises five regions (1. electron gun, 2. gas trap, 3. pulse-controller, 4. scattering chamber, and 5. analyser-detector region) which are surrounded by solenoids that apply independent magnetic fields to each region. These regions, differentially pumped by means of three turbomolecular pumps, are connected by small orifices $(1.5 \mathrm{~mm})$ in order to create a well-defined region of constant pressure in both the gas trap 
and the scattering chamber. The background pressure in the electron gun and analyser-detector regions is typically of the order of $10^{-8}$ Torr. In the pulse-controller region, the background pressure is higher $\left(10^{-6}\right.$ Torr $)$ since the typical nitrogen $\left(\mathrm{N}_{2}\right)$ pressure inside the gas trap is $6 \times 10^{-2}$ Torr. The electron beam, originating from the electron gun with a typical resolution $\Delta E=500 \mathrm{meV}$ (FWHM) passes through the $\mathrm{N}_{2}$ gas trap where electrons are forced to lose some of their initial kinetic energy, due to excitation of vibrational and discrete electronic states, to finally acquire a given set energy. In this process, the initial energy beam spread is also reduced [17] down to 160-350 meV, depending on the incident energy. Next, the beam emerging from the gas trap is pulsed with a typical time duration of $50 \mathrm{~ms}$ and a frequency of $10 \mathrm{~Hz}$. The electron beam is then transported to the scattering chamber, where the target molecule (sevoflurane here) is admitted via a variable leak valve. Note that the pressure in the scattering chamber is measured by a Baratron capacitance manometer. After passing into the analyser-detector region, electrons are energy-selected by a retarding potential analyser (RPA). Only electrons with a parallel component of kinetic energy higher than the RPA potential energy barrier may reach the detector. The detection system is operated in a single-pulse counting mode and the MCP anode is positively biased with $\sim 2 \mathrm{kV}$. The current pulses collected at the anode are converted into voltage pulses by means of a charge sensitive preamplifier. That output signal is subsequently amplified and converted into TTL signal through the use of a constant fraction discriminator. The resulting signals are finally transferred to the data acquisition system connected to a computer implemented with LabView software.

\section{B. Beam energy calibration and data validation}

Central to a valid measurement for the magnitude and shape of the total cross section, an accurate knowledge of the electron beam energy in the scattering chamber is needed. Such accurate information is also needed to benchmark the validity of the cross section measurements from the current setup. Thus, the present electron energy is calibrated using the well-known nitrogen resonance feature at $\sim 2.5 \mathrm{eV}[18,19,20]$ and our nitrogen total electron scattering cross section, in the energy range 1-20 eV, compares very favourably with the data from Szmytkowski et al. [18] to within $3 \%$ difference. Another important validation is to ensure that there are no significant changes in the absolute pressure value measured along the collision path length. In order to check that we have measured total cross sections (TCSs) from nitrogen, monitoring the gas pressure from two different manometers placed at both ends of the scattering chamber. No effect was observed. 


\section{Data acquisition methodology}

The current data acquisition methodology includes the following protocols:

I. Sevoflurane was supplied by Sigma-Aldrich with a quoted purity of $99 \%$, and we note that no further purification was undertaken by us. However, the sample was degased by repeated freezepump-thaw cycles prior to use in order to minimise any impurities.

II. Before sample admission to the scattering chamber, the electron energy resolution was further improved by setting optimal magnetic fields in each section of the experimental apparatus followed by a reduction of $\sim 75 \%$ of the transmitted intensity. In the latter case this was achieved by selecting a proper retarding potential value [12].

III. With the purpose of minimizing any possible multiple scattering effects, a convenient range of target gas pressures, during the attenuation measurements, was determined. In the present case we obtained the optimal pressure region from 0.5 to $6.0 \mathrm{mTorr}$. Note that for each energy, we have recorded at least three scans to achieve a statistical reproducibility $\leq 5 \%$.

IV. The total electron scattering cross sections are then obtained for each energy by using the BeerLambert attenuation law:

$$
I=I_{0} e^{-n l \sigma_{T}}=I_{0} e^{-p l \sigma_{e x p} / k T},
$$

where $I_{0}$ is the intensity without attenuation, $\sigma_{\text {exp }}$ is the experimental total scattering cross section, $n$ is the density of the target gas, $p$ is the partial pressure of the sample, $l$ is the collision path length (40 mm), $k$ is the Boltzmann constant, and $T$ is the gas temperature (K). In Fig. 3 we plot the typical attenuation curves for $E=1,3,13,30,70$ and $100 \mathrm{eV}$ electron impact together with their exponential fit curves. Note that the excellent fit to the data, at each energy, confirms that multiple scattering effects were minimised in this study. The corresponding slopes provide directly the experimental total scattering cross section, $\sigma_{\text {exp }}$.

\section{Experimental uncertainties}

Systematic and random errors include contributions from the uncertainties in the collision path length, the sample gas pressure measurements, the attenuation curve fit function and the standard deviation from the different sets of measurements at the same energy (which comprise any filament and temperature instability during the measurements). Combining the aforementioned factors, a 
general experimental uncertainty in the range $1.0-4.7 \%$ is obtained. In addition, an inherent systematic error of the present experimental technique is linked to the energy resolution which is related with the angular acceptance [12,15,16], entailing "missing angles" in the forward and backward scattering directions. The angular resolution $\left(\Delta \Theta^{\circ}\right)$ can be calculated from the energy resolution $(\Delta \mathrm{E})$ as:

$$
\Delta \theta^{\circ}=\arcsin \sqrt{\frac{\Delta E}{E}}
$$

The method used to obtain the energy resolution is reported in detail in refs. $[12,16]$. In the present experiment the energy resolution and the detector angular acceptance are $\Delta \mathrm{E} \leq 0.35 \mathrm{eV}$ and $\Delta \Theta \leq$ $26.60^{\circ}$, although we note that optimum values of $0.16 \mathrm{eV}$ and $2.0^{\circ}$ have been obtained here (see Table I).

\section{E. Theoretical method}

In order to further our knowledge on the electron scattering process from sevoflurane, and help to interpret the experimental data, we have used the IAM-SCAR+I method (independent atom model (IAM) applying the screened additivity rule (SCAR) with interferences terms included (I)). This method has been described in detail in previous publications [13-16,21,22]. Briefly, the molecular target is described as an aggregate of its individual atoms (i.e. C, H, F and $\mathrm{O}$ in this case). Each atomic target is represented by an ab initio interacting complex optical potential given by:

$$
V_{o p t}(\vec{r})=V_{R}(\vec{r})+i V_{a b s}(\vec{r})
$$

In Eq. (3), the real part accounts for elastic scattering while the imaginary part represents the inelastic processes which are considered as the 'absorption part' following the procedure of Staszewska et al. [23]. The real part is divided into three terms that include:

$$
V_{R}(\vec{r})=V_{s}(\vec{r})+V_{e x}(\vec{r})+V_{p o l}(\vec{r}),
$$

where $V_{\text {s }}$ represents a static term derived from a Hartree-Fock calculation of the atomic charge distribution [24], $V_{\text {ex }}$ an exchange term to account for the indistinguishability of the incident and target electrons [25] and $V_{\mathrm{pol}}$ a long-range polarization term [26].

The molecular cross sections are obtained from the atomic data by the screening corrected additivity rule (SCAR) procedure [27], incorporating interference (I) term corrections [14], by summing all the relevant atomic amplitudes, including the phase coefficients. In this approach we 
obtain the molecular differential scattering cross section (DCS), which integrated over all the scattered electron angular range gives the integral scattering cross section (ICS). Moreover, by taking the sum of the ICS for all open channels (elastic ICS and inelastic ICS) the TCS is obtained. Note that we do not include at this stage any contribution from vibrational and rotational excitation processes. However, for polar molecules, as is the case of sevoflurane, with a permanent dipole moment of 2.27 D [7], the rotational driven cross section (dipole-excitation cross sections $(+\mathrm{R})$ ) is obtained [16] and added to the IAM-SCAR+I TCS [28].

\section{Results and discussion}

In table I we present the experimental total electron scattering cross section data from sevoflurane, as a function of the electron energy, together with the estimated uncertainties, and energy and angular resolutions. Note that in general our poorer angular resolutions correspond to our lower electron impact energies, even at better energy resolutions. To perform a valid comparison between the experimental data and the calculated TCSs, the angular acceptance is interpreted as corresponding to the so-called missing angles in the forward and backward directions, which results in relatively lower measured TCS values [29]. Hence, the DCSs obtained from the IAMSCAR $+\mathrm{I}+\mathrm{R}$ computations have to be integrated over the range $\left[\Delta \Theta^{\circ}, 180^{\circ}-\Delta \Theta^{\circ}\right]$ with those results being subtracted from the IAM-SCAR+I+R TCS to enable a more valid comparison to our measured results. The tabulated TCS values from this procedure are given in table II, and we denote them as our IAM-SCAR+I+R- $\Delta \Theta$ TCS.

Figure 4 depicts the present experimental results on the total cross section together with our $a b$ initio calculated TCS with (IAM-SCAR+I+R) and without (IAM-SCAR+I) rotational contributions as well as those accounting for the missing angles, i.e. our (IAM-SCAR+I+R- $\Delta \Theta$ ) TCS. The uncertainty limits of both our experimental, and calculated IAM-SCAR+I+R- $\triangle \Theta$ TCS data have also been included in this figure.

At this point of the discussion it is worth noting that the associated uncertainties from the IAM$\mathrm{SCAR}+\mathrm{I}+\mathrm{R}-\Delta \Theta$ TCS calculation are to within $10 \%$, which is reasonable for electron impact energies > $20 \mathrm{eV}$ (our typical calculation validity range), since at lower energies (<20 eV), inherent limitations of the theoretical method may affect the results. The $10 \%$ uncertainty limit assigned to our calculation for energies above $20 \mathrm{eV}$ is supported by comparisons with accurate experimental data from similar molecules (see Ref. [15] and references therein). Another 
interesting aspect is the good agreement between the experimental data and the IAM-SCAR+I+R$\Delta \Theta$ TCS for electron energies $>40 \mathrm{eV}$, where $\mathrm{a}<13 \%$ difference is observed. However, for lower electron impact energies $(<40 \mathrm{eV})$, this discrepancy increases $(>14 \%)$, being particularly significant for $E<4 \mathrm{eV}$. Nonetheless, it is fair to say that both these sets of results agree reasonably well to within the combined uncertainty limits for electron impact energies above about $20 \mathrm{eV}$. The importance of considering the angular acceptance, when comparing the IAM-SCAR+I+R and the IAM-SCAR $+\mathrm{I}+\mathrm{R}-\Delta \Theta$ TCS results (see Fig. 4), is in support of the procedure established to account for the missing angles contribution. In addition, a closer inspection of Fig. 4 reveals some weak features visible between 5 and $10 \mathrm{eV}$ and a broad hump centered within 30-50 eV which is the typical structure for fluorine rich targets [30]. Since there is currently no other available data in the literature to compare against for electron scattering from sevoflurane, this latter observation remains speculative.

\section{Conclusions}

The present work provides for the first time total electron scattering cross section data from sevoflurane at incident energies in the range 1-300 eV, using a recently developed and implemented experimental setup [12] that makes use of a strong axial magnetic field. The excellent agreement between the measured total cross sections and the ab initio calculations (typically above $20 \mathrm{eV}$ ), considering both the angular limitation in the measurements and the validity range of the calculations, suggests that the present results form a good basis for assembling a cross section data base for modelling studies on electron interactions with sevoflurane [31]. Certainly, the work foreshadowed in Lange et al. [11] will further add to that cross section data base, providing crucial further information for understanding its chemical and biological activity, for this important molecule.

\section{Acknowledgments}

Authors acknowledge partial financial support from the Spanish Ministerio de Economia $y$ Competitividad (Project No. FIS2016-80440). F.F.S. also acknowledges the Portuguese National Funding Agency FCT through Researcher Contract No. IF-FCT IF/00380/2014, and, together with P.L.V., the Research Grant No. UID/FIS/00068/2013. Authors also acknowledge Prof. M. J. Brunger and Dr. L. Campbell their valuable help in discussing results and editing procedures. 


\section{References}

[1] R. G. Eckenhoff and J. S. Johansson, Pharmacol. Rev. 49, 343-367, 1997

[2] R. Pidikiti, T. Zhang, K. M. G. Mallela, M. Shamim, K. S. Reddy, and J. S. Johansson, Int. Congr. Ser. 1283, 155-159, 2005

[3] P. Tang, V. Simplaceanu, and Y. Xu, Biophys. J. 76, 2346-2350, 1999

[4] P. Tang, R. G. Eckenhoff, and Y. Xu, Biophys. J. 78, 1804-1809, 2000

[5] R. M. Jones, Br. J. Anaesth. 65, 527-536, 1990

[6] P. E. I. Tang, I. Zubryzcki, and Y. A. N. Xu, J. Comput. Chem. 22, 436-444, 2001.

[7] A. Lesarri, A. Vega-Toribio, R. D. Suenram, D. J. Brugh, and J.-U. Grabow, Phys. Chem. Chem. Phys. $12,9624,2010$

[8] J. J. J. Dom et al., Phys. Chem. Chem. Phys. 13, 14142, 2011

[9] T. X. Cui, V. Bondarenko, D. J. Ma, C. Canlas, N. R. Brandon, J. S. Johansson, Y. Xu and P. Tang, Biophys. J. 94, 4464-4472, 2008

[10] T. Langbein, H. Sonntag, D. Trapp, A. Hoffmann, W. Malms, E.-P. Röth, V. Mörs, and R. Zellner, Br. J. Anaesth. 82, 66-73, 1999

[11] E. Lange, A. M. Lozano, F. Blanco, M. H. F. Bettega, G. García, P. Limão-Vieira, and F. Ferreira da Silva, in preparation, 2018

[12] A. I. Lozano, J. C. Oller, K. Krupa, P. Limão-Vieira, F. Blanco, A. Muñoz, R. Colmenares, and G. García, Rev. Sci. Instrum., in press, 2018

[13] F. Blanco, J. Rosada, A. Illana, and G. García, Phys. Lett. A 374, 4420, 2010

[14] F. Blanco, L. Ellis-Gibbings, and G. García, Chem. Phys. Lett. 645, 71, 2016

[15] A. Traore Dubuis, A. Verkhovtsev, L. Ellis-Gibbings, K. Krupa, F. Blanco, D. B. Jones, M. J. Brunger, and G. García, J. Chem. Phys. 147, 054301, 2017

[16] M. C. Fuss, A. G. Sanz, F. Blanco, J. C. Oller, P. Limão-Vieira, M. J. Brunger, and G. García, Phys. Rev. A. 88, 042702, 2013

[17] J. P. Sullivan, S. J. Gilbert, J. P. Marler, R. G Greaves, S. J. Buckman, and C. M. Surko, Phys. Rev. A. $66,042708,2002$

[18] C. Szmytkowski, K. Maciag, and G. Karwasz, Phys. Scripta 54, 271-280, 1996

[19] M. J. Brunger and S. J. Buckman, Phys. Repts. 357, 215-458, 2002

[20] K. R. Hoffman et al., Phys. Rev. A 25, 1393-1403, 1982

[21] A. I. Lozano, J. C. Oller, D. B. Jones, R. F. da Costa, M. T. do N. Varella, M. H. F. Bettega, F. Ferreira da Silva, P. Limão-Vieira, M. A. P. Lima, R. D. White, M. J. Brunger, F. Blanco, A. Muñoz, and G. García, Phys. Chem. Chem. Phys., submitted, 2018

[22] D. B. Jones, F. Blanco, G. García, R. F. da Costa, F. Kossoski, M. T. do N. Varella, M. H. F. Bettega, M. A. P. Lima, R. D. White, and M. J. Brunger, J. Chem. Phys. 147, 244304, 2017

[23] G. Staszewska, D. W. Schwenke, D. Thirumalai, and D. G. Truhlar, Phys. Rev. A 28, 2740, 1983

[24] R. D. Cowan, The Theory of Atomic Structure and Spectra (University of California Press, London, 1981

[25] M. E. Riley and D. G. Truhlar, J. Chem. Phys. 63, 2182, 1975

[26] X. Zhang, J. Sun, and Y. Liu, J. Phys. B: At. Mol. Opt. Phys. 25, 1893, 1992

[27] F. Blanco and G. García, Phys. Lett. A 330, 230, 2004

[28] A. Zecca, L. Chiari, G. García, F. Blanco, E. Trainotti, and M. J. Brunger, J. Phys. B 43, 215204, 2010

[29] M. J. Brunger, S. J. Buckman, and K. Ratnavelu, J. Phys. Chem. Ref. Data 46, 023102, 2017

[30] C. Szmytkowski, A. Domaracka, P. Mozejko, E. Ptasińska-Denga and S. Kwitnewski, J. Phys. B 38, $745,2005$.

[31] M. J. Brunger, Int. Rev. Phys. Chem. 36, 333-376, 2017 


\section{Figure captions}

Fig. 1. A schematic representation of the molecular structure of sevoflurane (colour online). Figure drawn using the Jmol software.

Fig. 2. Schematic diagram of the present experimental configuration: (1) electron gun, (2) gas trap, (3) pulse-controller, (4) scattering chamber, (5) analyser-detector, (6) nitrogen inlet, (7) gas target inlet, (8) cooling water inlet/outlet, (9) water jacket, (10) scattering cell and scattering chamber focusing electrodes, (11) various transmission grids, $\left(\mathrm{P}_{1}, \mathrm{P}_{2}, \mathrm{P}_{3}\right)$ turbomolecular pumps, (RPA) retarding potential analyser, (MCP) dual micro-channel-plate assembly, (B $\left.\mathrm{B}_{\mathrm{eg}}, \mathrm{B}_{\mathrm{GT}}, \mathrm{B}_{\mathrm{P}}, \mathrm{B}_{\mathrm{SC}}, \mathrm{B}_{\mathrm{AD}}\right)$ magnetic fields in the electron gun, gas trap, pulse-controller/interface chamber, scattering chamber and detector area, respectively. Note that this figure is not drawn to scale (colour online).

Fig. 3. Representative attenuation curves with their corresponding exponential fit functions (colour online). See also legend on figure.

Fig. 4. Experimental total electron scattering cross sections from sevoflurane compared with our ab initio TCS calculations with (IAM-SCAR+I+R) and without (IAM-SCAR+I) rotations. The square points represent the integrated values from our IAM-SCAR+I+R calculation, over the range $\left[\Delta \Theta^{\circ}, 180^{\circ}-\Delta \Theta^{\circ}\right]$ (colour online), that accounts for the missing angles in the measured data. See also legend in figure and text.

\section{Table captions}

Table I. Experimental total electron scattering cross section data and error estimates from sevoflurane. Also shown are the relevant energy resolutions and "missing angle" ranges for each incident electron energy.

Table II. Total cross sections obtained from our IAM-SCAR+I+R calculations, but corrected for the missing angles over the angular range $\left[\Delta \Theta^{\circ}, 180^{\circ}-\Delta \Theta^{\circ}\right]$ in order to better correspond to our measured data. 
Fig. 1. A schematic representation of the molecular structure of sevoflurane (colour online). Figure drawn using the Jmol software.

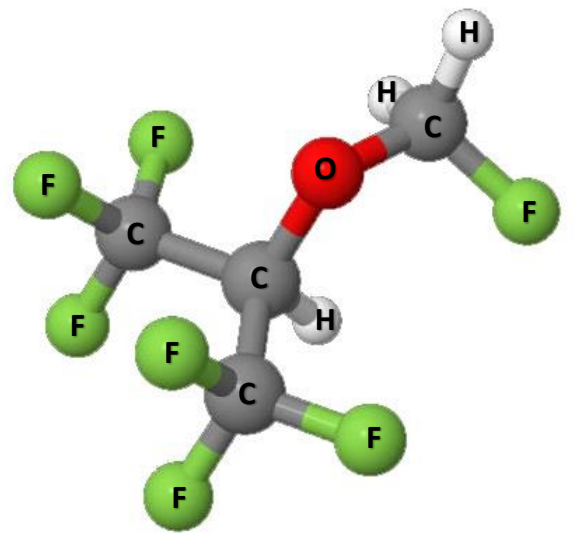


Fig. 2. Schematic diagram of the present experimental configuration: (1) electron gun, (2) gas trap, (3) pulse-controller, (4) scattering chamber, (5) analyser-detector, (6) nitrogen inlet, (7) gas target inlet, (8) cooling water inlet/outlet, (9) water jacket, (10) scattering cell and scattering chamber focusing electrodes, (11) various transmission grids, $\left(\mathrm{P}_{1}, \mathrm{P}_{2}, \mathrm{P}_{3}\right)$ turbomolecular pumps, (RPA) retarding potential analyser, (MCP) dual micro-channel-plate assembly, (Beg, $\left.\mathrm{B}_{\mathrm{GT}}, \mathrm{B}_{\mathrm{P}}, \mathrm{B}_{\mathrm{SC}}, \mathrm{B}_{\mathrm{AD}}\right)$ magnetic fields in the electron gun, gas trap, pulse-controller/interface chamber, scattering chamber and detector area, respectively. Note that this figure is not drawn to scale (colour online).

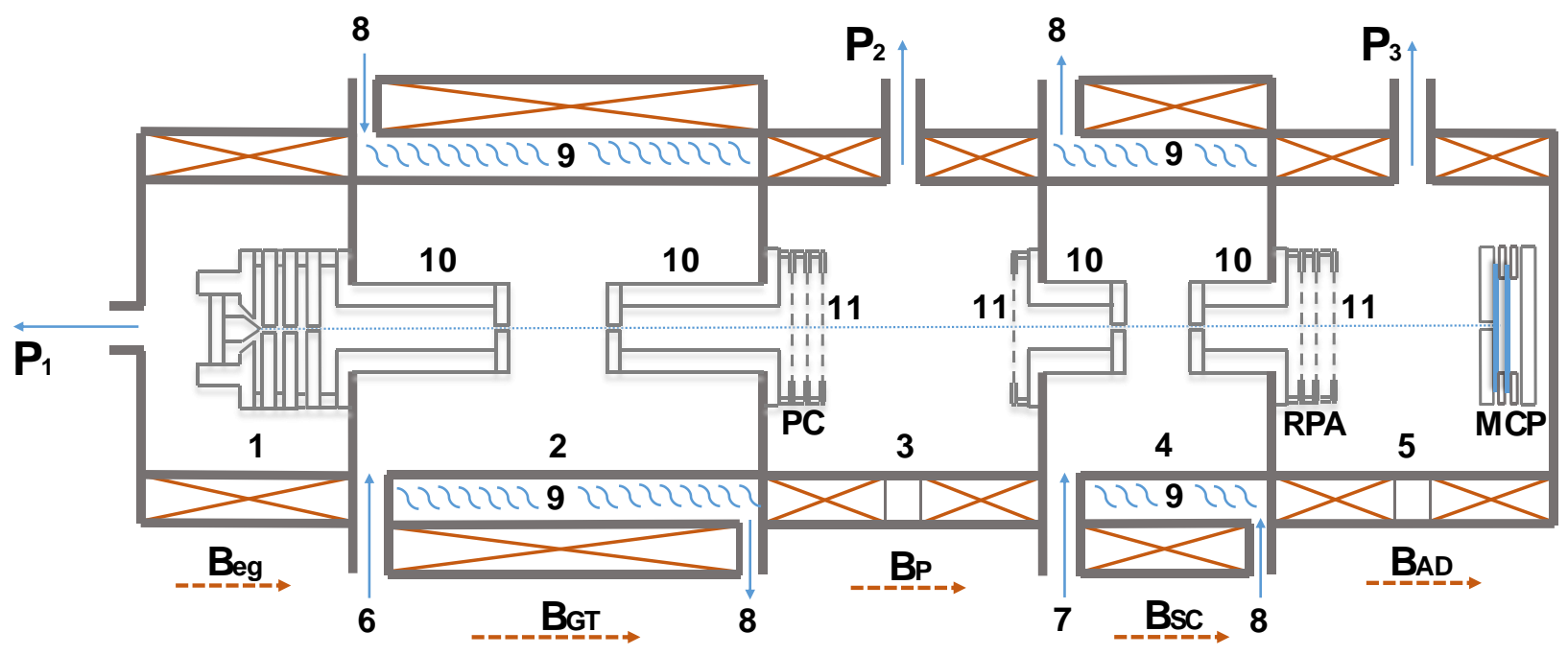


Fig. 3. Representative attenuation curves with their corresponding exponential fit functions (colour online). See also legend on figure.

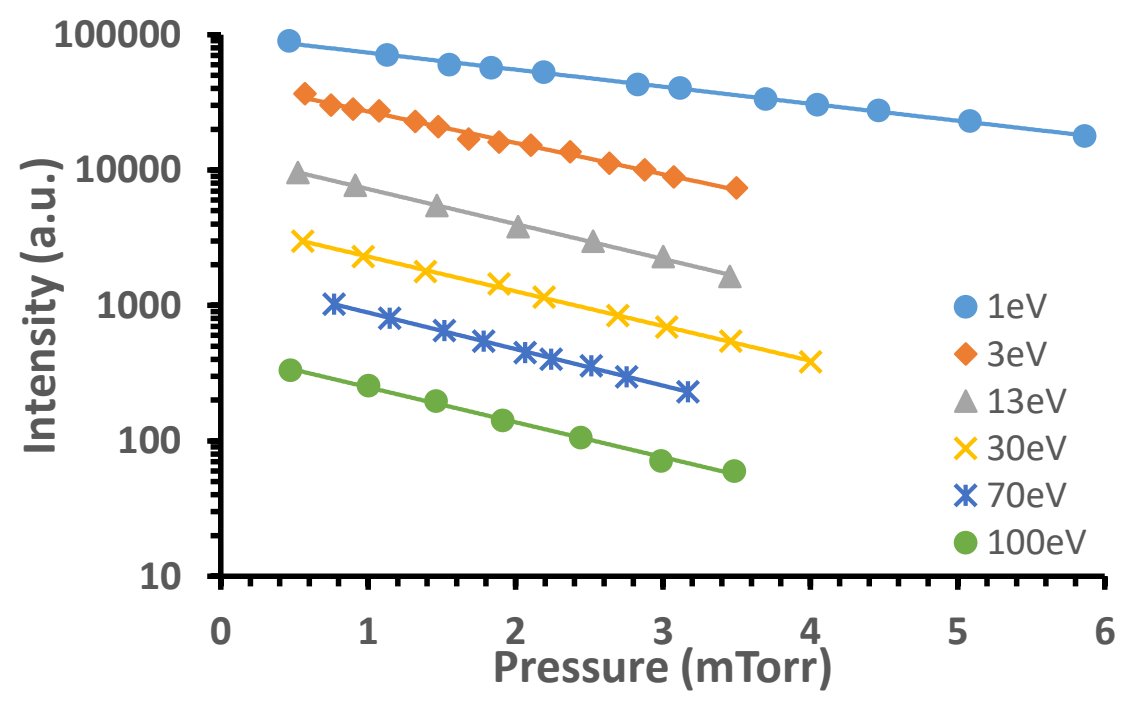


Fig. 4. Experimental total electron scattering cross sections from sevoflurane compared with our ab initio TCS calculations with (IAM-SCAR+I+R) and without (IAM-SCAR+I) rotations. The square points represent the integrated values from our IAM-SCAR+I+R calculation, over the range $\left[\Delta \Theta^{\circ}, 180^{\circ}-\Delta \Theta^{\circ}\right]$ (colour online), that accounts for the missing angles in the measured data. See also legend in figure and text.

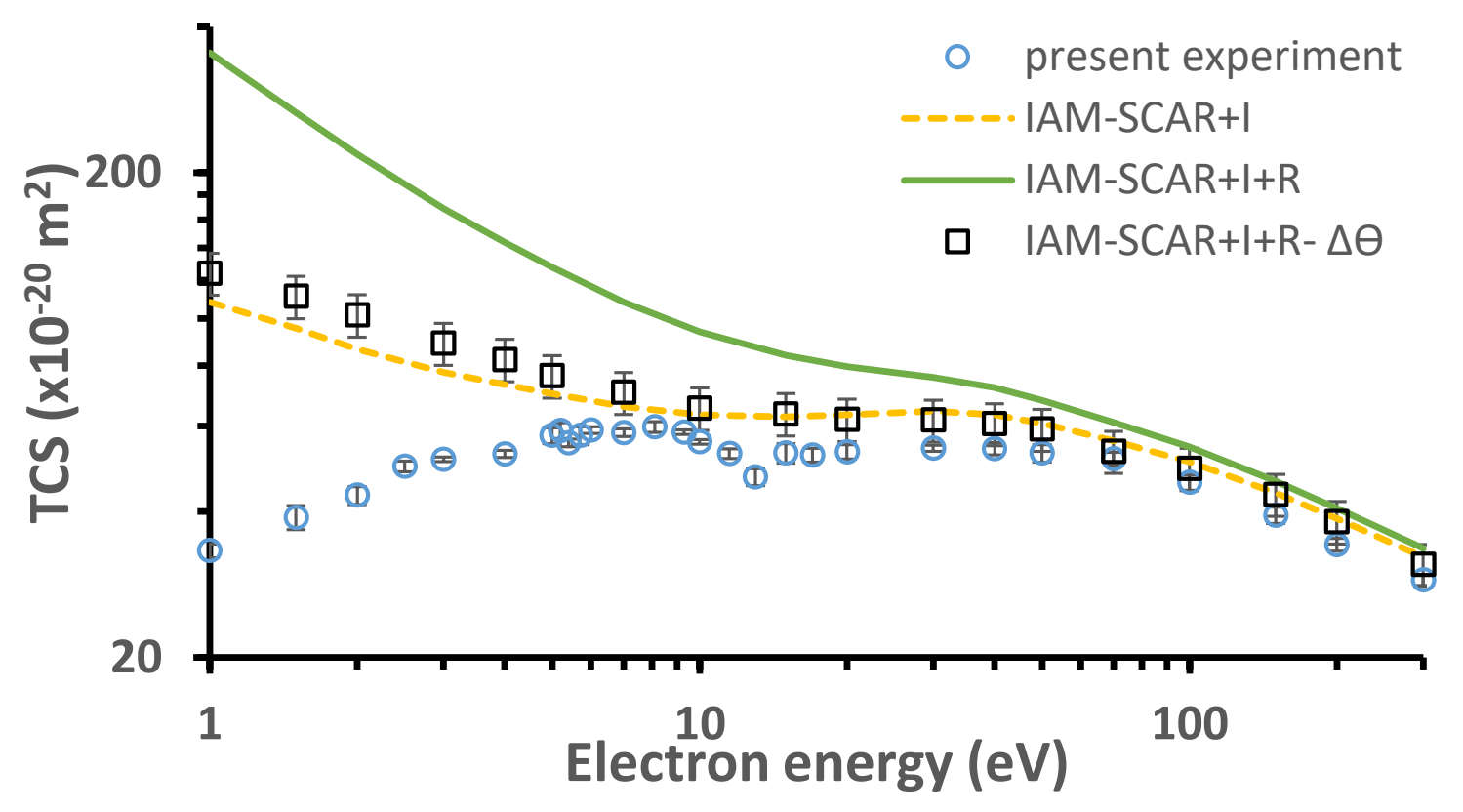


Table I. Experimental total electron scattering cross section data and error estimates from sevoflurane. Also shown are the relevant energy resolutions and "missing angle" ranges for each incident electron energy.

\begin{tabular}{|c|c|c|c|c|c|}
\hline Energy $(\mathrm{eV})$ & $\sigma_{\exp }\left(10^{-20} \mathrm{~m}^{2}\right)$ & $\begin{array}{c}\text { Statistical } \\
\text { uncertainty } \\
(\%)\end{array}$ & $\begin{array}{c}\text { Absolute total } \\
\text { uncertainty } \\
\text { limit }( \pm)\end{array}$ & $\Delta \mathrm{E}(\mathrm{eV})$ & $\Delta \Theta(\operatorname{deg})$ \\
\hline 1.0 & 33.2 & 3.2 & 1.1 & 0.20 & 26.6 \\
\hline 1.5 & 39.1 & 3.1 & 1.2 & 0.19 & 20.8 \\
\hline 2.0 & 43.2 & 4.3 & 1.8 & 0.18 & 17.4 \\
\hline 2.5 & 49.55 & 2.5 & 1.2 & 0.16 & 14.7 \\
\hline 3.0 & 51.24 & 1.0 & 0.5 & 0.18 & 14.2 \\
\hline 4.0 & 52.6 & 1.7 & 0.9 & 0.17 & 11.9 \\
\hline 5.0 & 57.4 & 3.8 & 2.2 & 0.22 & 12.1 \\
\hline 5.2 & 58.78 & 3.8 & 2.2 & 0.24 & 12.4 \\
\hline 5.4 & 55.4 & 1.8 & 1.0 & 0.23 & 11.9 \\
\hline 5.7 & 57.4 & 4.3 & 2.5 & 0.21 & 11.1 \\
\hline 6.0 & 58.9 & 1.7 & 1.0 & 0.19 & 10.3 \\
\hline 7.0 & 58.2 & 1.8 & 1.1 & 0.22 & 10.2 \\
\hline 8.1 & 59.8 & 2.5 & 1.5 & 0.23 & 9.7 \\
\hline 9.3 & 58.3 & 1.1 & 0.7 & 0.25 & 9.4 \\
\hline 10 & 55.7 & 1.1 & 0.6 & 0.23 & 8.7 \\
\hline 11.5 & 52.7 & 2.3 & 1.2 & 0.26 & 8.6 \\
\hline 13 & 47.1 & 4.0 & 1.9 & 0.25 & 8.0 \\
\hline 15 & 52.8 & 4.7 & 2.5 & 0.24 & 7.3 \\
\hline 17 & 52.3 & 3.2 & 1.7 & 0.24 & 6.8 \\
\hline 20 & 53.1 & 3.4 & 1.8 & 0.30 & 7.0 \\
\hline 30 & 54.0 & 1.5 & 0.8 & 0.30 & 5.7 \\
\hline 40 & 53.9 & 3.0 & 1.6 & 0.31 & 5.1 \\
\hline 50 & 52.8 & 4.1 & 2.2 & 0.29 & 4.4 \\
\hline 70 & 51.4 & 3.1 & 1.6 & 0.30 & 3.8 \\
\hline 100 & 45.9 & 3.8 & 1.8 & 0.23 & 2.7 \\
\hline 150 & 39.3 & 3.9 & 1.6 & 0.25 & 2.3 \\
\hline 200 & 34.2 & 3.1 & 1.1 & 0.35 & 2.4 \\
\hline 300 & 28.9 & 2.6 & 0.8 & 0.35 & 2.0 \\
\hline
\end{tabular}


Table II. Total cross sections obtained from our IAM-SCAR+I+R calculations, but corrected for the ionising angles over the angular range $\left[\Delta \Theta^{\circ}, 180^{\circ}-\Delta \Theta^{\circ}\right]$ in order to better correspond to our measured data.

\begin{tabular}{|l|l|}
\hline Energy $(\mathrm{eV})$ & IAM-SCAR+I+R- $\Delta \Theta$ TCS $\left(10^{-20} \mathrm{~m}^{2}\right)$ \\
\hline 1.0 & 124.1 \\
\hline 1.5 & 111.1 \\
\hline 2.0 & 101.7 \\
\hline 3.0 & 89.0 \\
\hline 4.0 & 82.3 \\
\hline 5.0 & 76.2 \\
\hline 7.0 & 70.4 \\
\hline 10 & 65.4 \\
\hline 15 & 63.6 \\
\hline 20 & 62.0 \\
\hline 30 & 61.7 \\
\hline 40 & 60.7 \\
\hline 50 & 59.1 \\
\hline 70 & 53.2 \\
\hline 100 & 49.1 \\
\hline 150 & 43.4 \\
\hline 200 & 38.1 \\
\hline 300 & 31.1 \\
\hline
\end{tabular}

